This item was submitted to Loughborough's Research Repository by the author.

Items in Figshare are protected by copyright, with all rights reserved, unless otherwise indicated.

\title{
Investigating mechanical anisotropy and end-of-vector effect in laser-sintered nylon parts
}

PLEASE CITE THE PUBLISHED VERSION

PUBLISHER

(C) Professional Engineering Publishing

\section{LICENCE}

CC BY-NC-ND 4.0

\section{REPOSITORY RECORD}

Ajoku, Uzoma, Naguib Saleh, Neil Hopkinson, Richard J.M. Hague, and Poonjolai Erasenthiran. 2019. "Investigating Mechanical Anisotropy and End-of-vector Effect in Laser-sintered Nylon Parts". figshare. https://hdl.handle.net/2134/3562. 
This item was submitted to Loughborough's Institutional Repository by the author and is made available under the following Creative Commons Licence conditions.

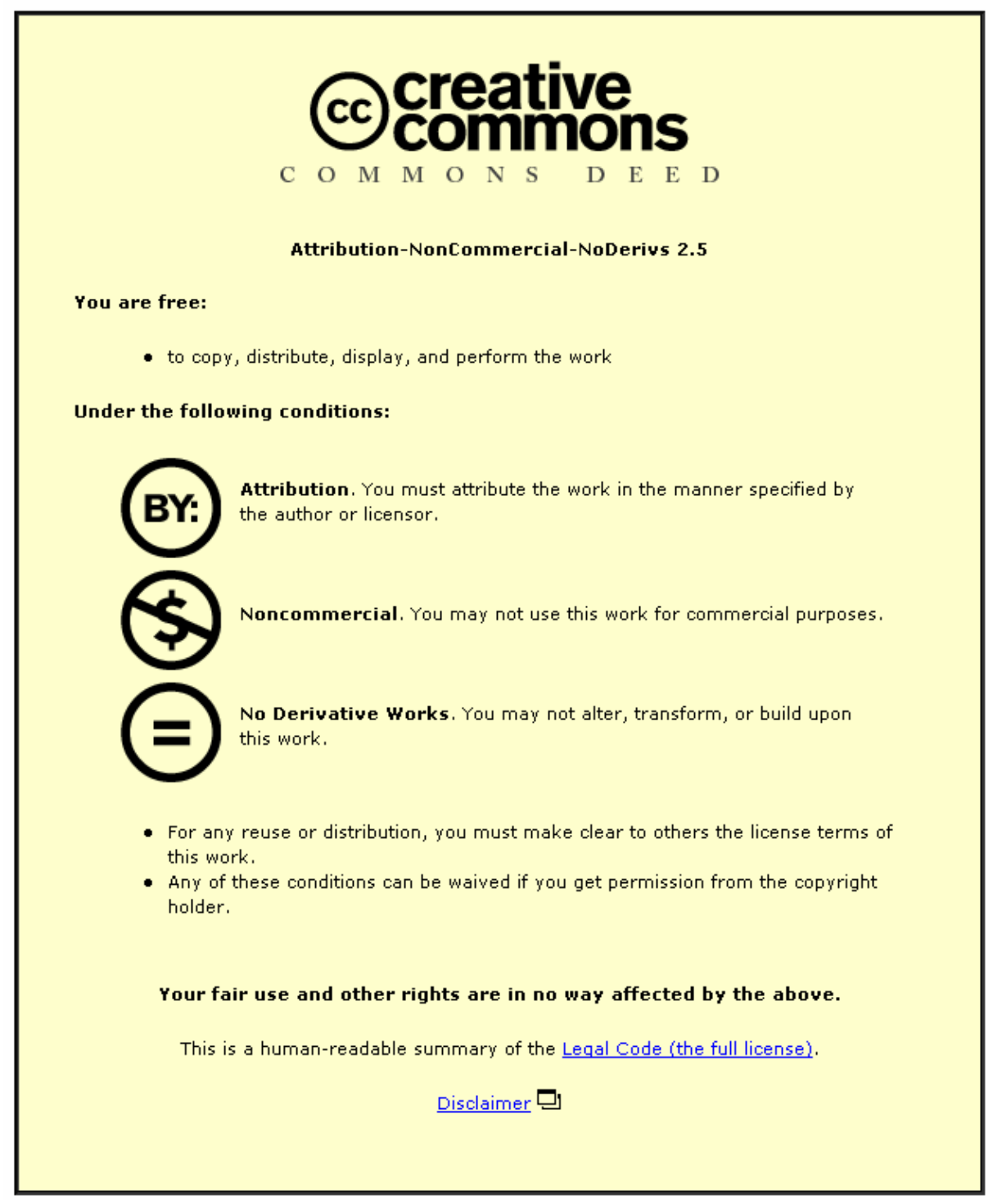

For the full text of this licence, please go to: http://creativecommons.org/licenses/by-nc-nd/2.5/ 


\title{
Investigating mechanical anisotropy and end-of-vector effect in laser-sintered nylon parts
}

\author{
U Ajoku*, N Saleh, N Hopkinson, R Hague, and P Erasenthiran \\ Wolfson School of Mechanical and Manufacturing Engineering, Loughborough University, Loughborough, UK
}

The manuscript was received on 4 January 2006 and was accepted after revision for publication on 10 March 2006.

DOI: $10.1243 / 09544054 J E M 537$

\begin{abstract}
A study investigating the effects of part-build orientation in the laser sintering process is presented. The investigation uses tensile, flexural, and compression testing methods to assess the changes in the mechanical properties of laser-sintered nylon-12 parts. The test parts were built in the $x, y$, and $z$ orientations with the $x$ axis parallel to the direction of the laser scanning, the $y$ axis perpendicular to the direction laser of scanning, and the $z$ axis in the direction of powder layers. The results from the tests show that the build orientation of the parts has an effect on the mechanical properties produced. The tensile tests show a maximum difference of 16 per cent and 11.2 per cent in strength and modulus respectively for parts built in the $x, y$, and $z$ axes. The flexural tests show a 9.4 per cent and 7 per cent maximum difference in strength and modulus respectively for the parts produced in the $x, y$, and $z$ axes. For the compressive tests, there is a 3.4 per cent and 13.4 per cent maximum difference in strength and modulus respectively for the parts produced in the $x, y$, and $z$ axes. A statistical analysis of the results obtained highlights the presence of anisotropy in tensile and compression parts owing to their build orientation in the laser sintering machine. The test parts built in the $x$ axis orientation showed the highest strength and modulus values while the parts built in the $z$ axis orientation showed poor strength and modulus values. However, this is not the case for the flexural test parts, which show the highest strength and modulus values are from those built in the $y$ axis orientation. Analysis has shown that this is due to the end-of-vector effect, which is most prominent in the $y$ axis orientation. This effect should always be considered during laser sintering, when mechanical integrity is vital.
\end{abstract}

Keywords: rapid prototyping (RP), laser sintering (LS), rapid manufacturing (RM), nylon-12, mechanical properties

\section{INTRODUCTION}

\subsection{Background}

Laser sintering (LS) is a rapid prototyping (RP) technology that uses a layer-based system to create complex three-dimensional objects. Components are built directly from three-dimensional computeraided design (CAD) files that have been converted into a STL file format. A range of materials in powder

*Corresponding author: Rapid Manufacturing Research Group, Wolfson School of Mechanical and Manufacturing Engineering, Loughborough University, Leicestershire LE11 3TU, UK. email: u.k.ajoku@lboro.ac.uk form, such as metals, ceramics, and polymers, are available for the LS process [1]. The LS process uses a $\mathrm{CO}_{2}$ laser to trace out and selectively sinter a layer of the part being produced from powder material, as shown in Fig. 1.

After a layer is scanned, a new layer of material is added and the process is repeated; this continues until the entire part is completed. Any material that is not sintered (loose powder) in and around the required component acts as support for the component. This is simply 'brushed off' when the part is completed and can be reused if necessary, as long as the correct ratio of virgin powder to used powder mix is achieved. There are, however, some thermal processing issues that should be 


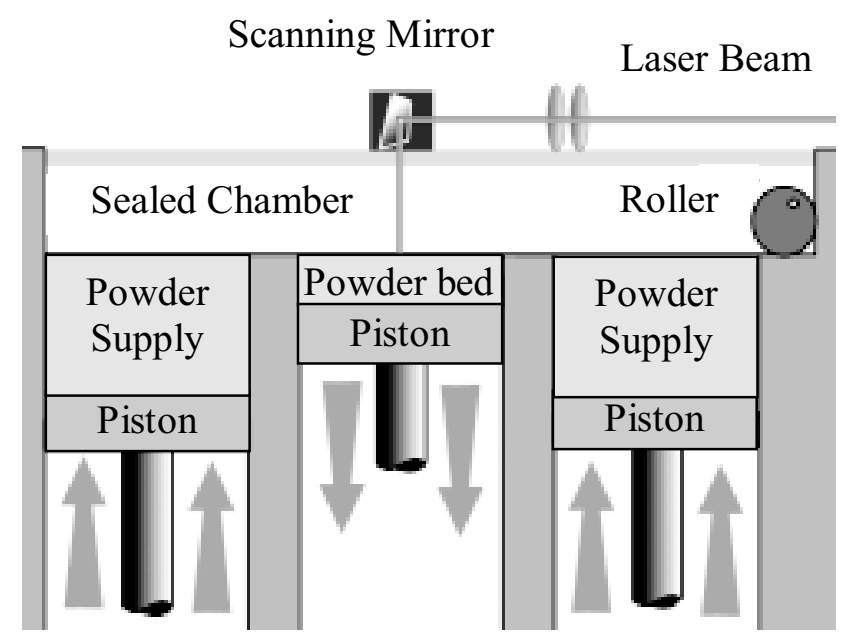

Fig. 1 Laser-sintering process [2]

addressed before using 'recycled' powder. In order to improve the finish on laser-sintered parts, post-processing techniques such as bead-blasting, sanding, and painting are employed. The advantages and disadvantages of the LS process have been discussed in detail by Grimm [3].

\subsection{The sintering process}

The sintering of polymers is defined as the formation of a homogeneous melt from the bonding of particles. This is considered a double-stage mechanism, involving powder sintering and pore removal [4]. This is also referred to as the growth of 'necks' and the equilibration of material properties within those necks [5]. The characteristic time for shape relaxation is governed by the competition between surface tension (surface area reduction) and viscous dissipation [6]. Surface tension is the driving force for sintering while the viscous forces must be overcome in order to allow powder coalescence or sintering. Ceramics, metals, and polymers all exhibit different sintering properties. In the LS of semicrystalline polymers, sintering occurs after the laser partly melts the powder and causes viscous flow to occur $[3,7]$.

Viscous sintering was first explored by Frenkel [8] who put forward a theory which states that the energy dissipated during the viscous flow is equal to the energy gained by the reduction in the surface area. Frenkel used an equation to describe the neck growth in a two-particle system, as shown in Fig. 2

$$
\left(\frac{x}{r}\right)^{2}=\frac{3 \sigma t}{2 r \eta_{0}}
$$

From equation (1), $x=$ radius of the neck, $r=$ radius of the sphere, $\sigma=$ surface tension of the material, $t=$ time needed for sintering, and $\eta_{\mathrm{o}}=$ melting viscosity.

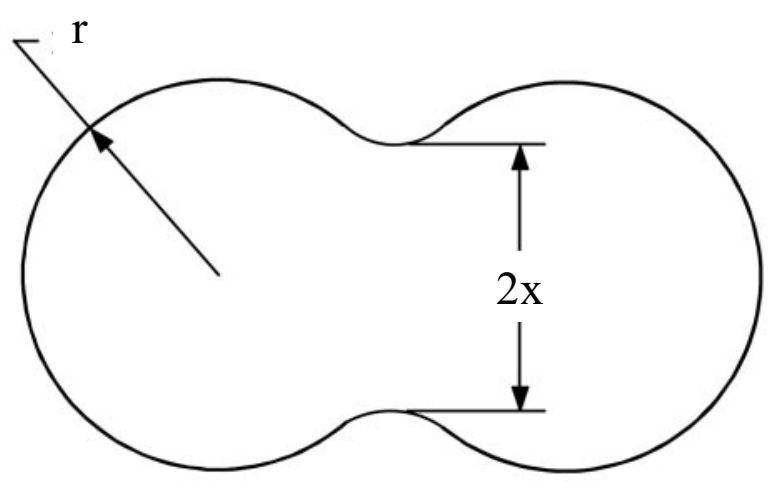

Fig. 2 Frenkel sintering model

Scherer $[\mathbf{9}, \mathbf{1 0}]$ took Frenkel's idea further by developing a model that described the sintering behaviour of a viscous glass preform. Scherer assumed that the surface energy reduction of the sintering powder drives the process through viscous mass flow dissipation. Scherer suggested that the glass powder consisted of an open-pore network of cylinders arranged cubically with the cylinder diameters equal to the particle diameter and the cylinder lengths proportional to the pore diameter in the low density range $(\Delta \leqslant 0.94)$. Scherer used a free strain term $(e)$ to describe the densification rate for viscous sintering using equations (2) and (3) below

$$
\begin{aligned}
& \frac{\partial e}{\partial t}=-\frac{M}{\eta_{\mathrm{o}}} \frac{(3 \pi)^{1 / 3}}{6} \frac{2-3 c x}{\sqrt[3]{x(1-c x)^{2}}} \quad \text { when } \Delta \leqslant 0.94 \\
& \frac{\partial e}{\partial t}=-\frac{M}{2 \eta_{\mathrm{o}}}\left(\frac{4 \pi}{3}\right)^{1 / 3}\left(\frac{1}{\Delta}-1\right)^{2 / 3} \quad \text { when } \Delta>0.94
\end{aligned}
$$

where $M=(\sigma / r)(3 / 4 \pi)^{1 / 3}$, constant $c=8 \sqrt{2} / 3 \pi$, $\eta_{\mathrm{o}}=$ viscosity, $\Delta=$ relative density defined as the ratio of the part density to the material theoretical density, $\sigma=$ surface tension, $\mathrm{r}=$ particle initial radius, and $x$ is defined as the ratio of the cylinder structure to the length of the cylinder.

The porosity, $\varepsilon=(1-\Delta)$ can be related to the free strain $e$ by

$$
\begin{aligned}
& \varepsilon=1-\left(1-\varepsilon_{o}\right) \exp (-3 e) \quad \text { when } \Delta \leqslant 0.94 \\
& \varepsilon=1-3 \pi x^{2}+8 \sqrt{2} x^{3} \quad \text { when } \Delta>0.94
\end{aligned}
$$

where $\varepsilon_{\mathrm{o}}=$ initial porosity when $\mathrm{t}=0$.

Another sintering model was developed by Sun et al. [11]. The model describes the stages which a spherical particle undergoes during the LS process (see Fig. 3). Each spherical particle of initial radius $a$, is contained within a cube of side length $2 x$. During sintering, the cube dimension of $2 x$ decreases while the sphere radius $r$ increases to maintain constancy of volume. 


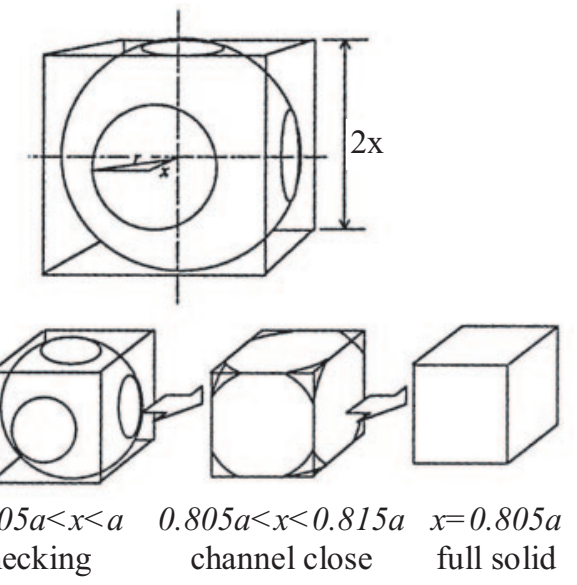

Fig. 3 The unit cell and densification process of a cubic pack structure [11]

A separation of the pore occurs when $r=\sqrt{2} x$ (when $x=0.815 a$ ). During this period, the sintering rate $\dot{x}$ is given by

$$
\dot{x}=-\frac{\pi \sigma a^{2}}{6 \eta_{\mathrm{o}} x^{3}}\left\{r-(1-\zeta) x+\left[x-\left(\zeta+\frac{1}{3}\right) r\right] \frac{9\left(x^{2}-r^{2}\right)}{18 r x-12 r^{2}}\right\}
$$

where $\zeta$ is a number between 0 and 1 associated with the probability of formation of sintering necks between particles and is also a function of the relative density. For small values of $\zeta$ less than 0.3, the parameter is proportional to the relative density according to $\zeta \approx(2 \rho-1) / 3$.

\subsection{Previously reported work on the properties of LS parts}

Although the LS process has been developed as an RP technology, it can be applied to other areas such as rapid tooling (RT) and rapid manufacturing (RM) [12-14]. With more manufacturers using the LS process for RM, it is vital that designers understand the properties of the materials that are being used. Much work has been focused on determining the most appropriate parameters for the LS process. Gibson and Shi [15] were able to show how the fabrication parameters of the LS process influenced the material properties of LS parts. This was achieved by carrying out experiments measuring the tensile strength and density of specimens that had been built in different orientations with different fabrication parameters. Zarringhalam and Hopkinson [16] showed that post-processing in the form of heat treatment can also improve the tensile and impact strengths of LS parts made from nylon12. Ho, Gibson, and Cheung [17] conducted experiments showing the effects of the energy density (ED) of a laser beam on polycarbonate powder. At low ED levels the density and tensile strength of spe- cimens increased with increasing ED. Although at ED levels above $0.09 \mathrm{Jmm}^{-2}$, degradation of the polycarbonate powder occurred and there was a reduction in density and tensile strength. Tontowi and Childs [18] showed that the powder-bed temperature affects the density of sintered parts. By keeping the ED constant, the powder-bed temperature was varied. At a high temperature of $182^{\circ} \mathrm{C}, 100$ per cent density was achieved, but at a lower temperature of $178^{\circ} \mathrm{C}$, the density reduced by 4 per cent.

\subsection{Anisotropy in LS-produced parts}

Anisotropy affects the quality of all LS-produced parts. Gibson and Shi [15] have investigated anisotropy in polymer parts made via the LS process. Tensile bars were built in a variety of orientations using 3D System's 'fine nylon' material on a commercial Sinterstation 2000 machine, and measured the parts built in the $z$ orientation as having the worst tensile strength because the applied force was in the layer direction. The parts built in the $x$ orientation showed a slightly higher average tensile strength value when compared with those built in the $y$ orientation because of a larger cross-sectional area. Although these results show anisotropy, the parameters used were different from the standard set because even the in-plane strength ( $x$ orientation) was below half the manufacturer's specification for this material.

\section{THE THEORY BEHIND RESULTS OBTAINED FROM PREVIOUS WORK}

\subsection{The LS process sequence and mechanical properties}

Previously discussed work on the sintering process in section 1.2 highlighted the role of temperature during the sintering process. Frenkel demonstrated this using equation (1), where higher temperatures lead to an increased necking radius between sintered particles $[\mathbf{8}]$. Increasing the necking radius encourages greater bonding between the particles and reduces pores. Scherer $[\mathbf{9}, \mathbf{1 0}]$ demonstrated this principle by showing that greater temperatures increased density by reducing pore diameter, while Sun et al. [11] showed that varying the temperature has a significant effect on the degree of porosity. These findings would suggest that the results obtained from previously conducted work on LS discussed in section 1.3 may be owing to the degree of bonding between particles and the subsequent bonding between layers.

During the LS process, the infrared laser sinters components layer by layer. As the laser scans a vector on a layer, the melted polymer powder 


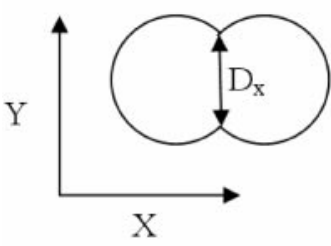

(a)

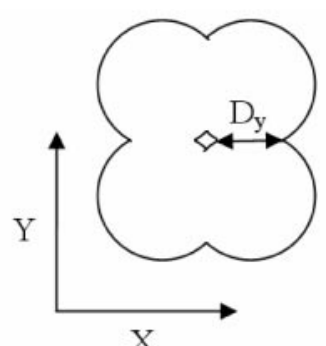

(b)

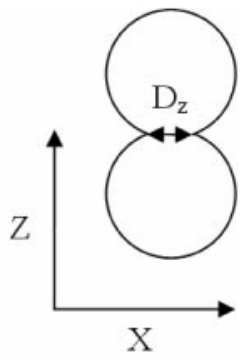

(c)

Fig. 4 Cross-section of particle-particle bonding in $x, y$, and $z$ axes $\left(D_{x}>D_{y}>D_{z}\right)$

particles bond together with a neck diameter $D_{x}$. This is a particle-particle bonding mechanism, as illustrated in Fig. 4(a). The laser then switches off and an increment occurs in the $y$ axis mirror to allow the laser to scan the next vector. The bonding between particles along the $x$ axis is much the same as with the previous vector shown in Fig. 4(a); however, particles in the previous vector will have cooled so that bonding between particles between vectors (in the $y$ axis) will be less than that in the $x$ axis (see Fig. 4(b)) with a smaller necking diameter $D_{y}$. When a layer has been completely sintered, a new layer of powder is applied and the particles in the previous layer will have cooled to such an extent that bonding between particles in different layers is less than that between particles in different vectors (see Fig. 4(c)) with an even smaller neck diameter, $D_{z}$.

In LS the laser scans across the whole twodimensional area, which consists of parts being built. The larger the two-dimensional scan area, the greater the time taken for the laser to restart scanning on a subsequent layer. As previously stated, when sintering large cross-sectional profiles, this can result in too much cool down, minimizing sintering between the layers. This leads to lower mechanical properties owing to the poor bonding between layers.

\subsection{The end-of-vector effect}

All components built on an LS machine are affected by what is known as the end-of-vector (EOV) effect. The EOV effect occurs owing to the degree of laser beam exposure at the start of a sinter scan on a part. A schematic of a laser exposure graph is shown in Fig. 5.

Figure 5 shows that as the laser begins to scan a line of powder, there is always an initial burst of energy, which stabilizes after a few milliseconds. At the end of each scan, the laser stops, the $y$ mirror moves and the process is repeated. By taking Fig. 5 and superimposing it on a cross-section of a part, the EOV effect is better illustrated (see Fig. 6.).

The short bursts of energy at the edges result in components being much denser at the edges than

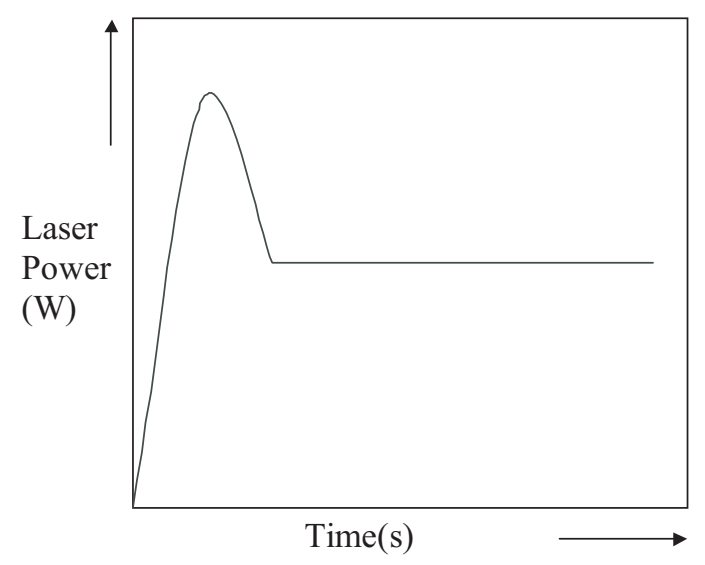

Fig. 5 Schematic diagram of laser exposure

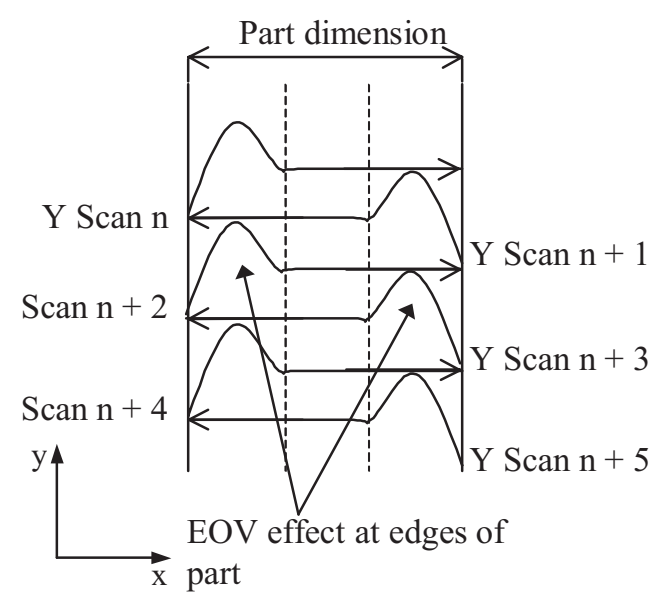

Fig. 6 EOV effect on a thin wall part

at the centres, thus improving the mechanical properties in thin sections where the $x$ dimension is small. Although the EOV effect always occurs, it is most prominent in parts that have small dimensions and are placed against the direction of laser scanning (i.e. perpendicular to the $x$ axis in the Vanguard SLS machine). It should also be noted that this phenomenon only occurs at the start of each vector and not, as the name suggests, at the end of each vector. 
Table 1 Sintering process parameters

\begin{tabular}{ll}
\hline Laser power & $11 \mathrm{~W}$ \\
Outline laser power & $5 \mathrm{~W}$ \\
Laser spot diameter & $0.46 \mathrm{~mm}$ \\
Laser scan spacing & $0.15 \mathrm{~mm}$ \\
Layer thickness & $0.1 \mathrm{~mm}$ \\
Laser scan speed & $5000 \mathrm{~mm} / \mathrm{s}$ \\
Laser scan strategy & $x$ direction only \\
Particle size & $58 \mu \mathrm{m}$ \\
Temperature of sintering room & $23^{\circ} \mathrm{C}$ \\
\hline
\end{tabular}

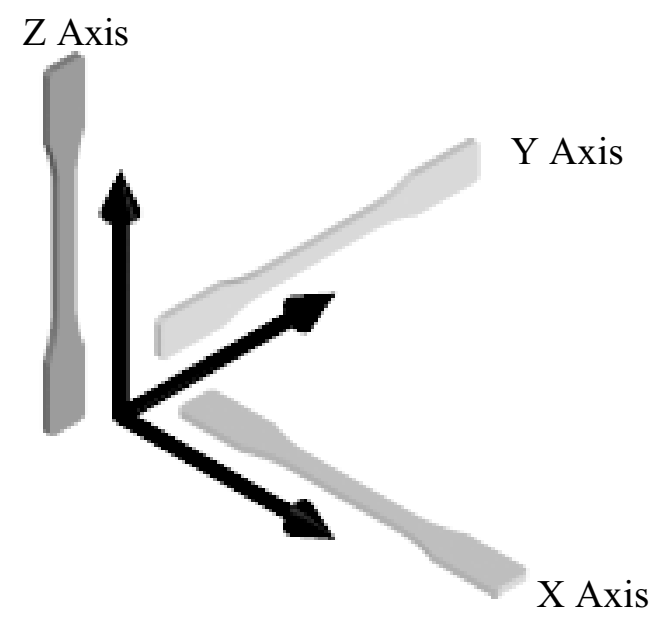

Fig. 7 Build orientation of test parts

This investigation looks at what effect the build orientation and EOV effect has on the mechanical properties of an LS part. For this study, a nylon-12 polymer material commercially known as Duraform PA was used. Duraform PA is currently one of the most commonly used materials in LS.

\section{METHODOLOGY}

\subsection{Equipment used}

All test specimens were built on a 3D Systems Vanguard SI laser sintering machine using virgin powder. The process parameters used were the standard manufacturer's specification, which was kept the same for all test parts. These are shown in Table 1.

Although there is currently a laser scan strategy for $x y$ scanning, this paper investigates the degree of anisotropy when using $x$ scanning only and whether investment in upgrades is of any value. A Zwick Universal testing machine with a $10 \mathrm{kN}$ load cell was used to conduct the mechanical property tests. All tests were conducted in a temperature and humidity controlled room, which was set at $20^{\circ} \mathrm{C}$ and 40 per cent relative humidity.

\subsection{Test specimens}

Figure 7 shows the $x, y$, and $z$ axis build orientations of the LS tensile test parts used for this research. The same orientations were used for the flexural and compression test parts. The orientation of the test parts was chosen to assess the variations in mechanical properties between the $x, y$, and $z$ directions and thereby understand the degree of anisotropy inherent in the process. By placing the test parts on their 'narrow side' for the $y$ and $z$ build orientation, the EOV effect will be more prominent within the LS test parts. A total of 15 test samples were built for each test with five samples in each orientation. The tensile, flexural, and compression tests were adherent to the ISO standards [19-21] and were conducted to observe what effect the sample orientation had on the test results. A test speed of $1 \mathrm{~mm} / \mathrm{min}$ was used to determine the tensile modulus $\left(E_{\mathrm{T}}\right)$ after which the rate was increased to $5 \mathrm{~mm} / \mathrm{min}$ to measure the ultimate tensile strength (UTS), along with elongation at break $(\varepsilon)$. The flexural test speed rate was also $1 \mathrm{~mm} / \mathrm{min}$ for the flexural modulus $\left(E_{\mathrm{F}}\right)$ while the rate was increased to $5 \mathrm{~mm} / \mathrm{min}$ to determine its ultimate flexural strength (UFS). For the compression tests, a test rate of $1 \mathrm{~mm} / \mathrm{min}$ was used to determine the compressive modulus $\left(E_{\mathrm{C}}\right)$ while a test rate of $5 \mathrm{~mm} / \mathrm{min}$ was used for the determination of the compressive strength $\left(\sigma_{\mathrm{c}}\right)$.

Based on the build orientation in Fig. 7, the total two-dimensional scanned cross-sectional area for each test part was determined in order to establish whether the size of the area scanned would affect the mechanical properties obtained. These were derived from the sum total of the cross-sectional area of the test parts in the $x, y$, and $z$ axes. The tensile, flexural, compression modulus, and compression strength parts respectively showed a 3 per cent, 0.9 per cent, 0.6 per cent, and 0.1 per cent total twodimensional scanned cross-sectional area. Based on the discussion in section 2.1, these were deemed insufficient adversely to affect mechanical properties.

\section{RESULTS}

The results of the mechanical properties investigated are shown in Table 2. This shows average results from 15 test parts built in each orientation (five tensile, five flexural, and five compression).

\subsection{Tensile properties}

Figures 8 to 10 show the UTS, $E_{\mathrm{T}}$, and $\varepsilon$ results obtained, against their build orientations. The 
Table 2 Properties of test parts in different build orientations

\begin{tabular}{|c|c|c|c|c|}
\hline $\begin{array}{l}\text { Mechanical } \\
\text { property }\end{array}$ & $x$ axis & $y$ axis & $z$ axis & $\begin{array}{l}\text { Maximum } \\
\text { difference (\%) }\end{array}$ \\
\hline \multicolumn{5}{|l|}{ Tensile } \\
\hline \multicolumn{5}{|c|}{ UTS (MPa) } \\
\hline Ave. & 48.7 & 44.7 & 40.9 & \multirow[t]{3}{*}{16.0} \\
\hline Max. & 50.4 & 47.9 & 42 & \\
\hline Min. & 46.9 & 38.5 & 39.9 & \\
\hline \multicolumn{5}{|l|}{$E_{\mathrm{T}}(\mathrm{MPa})$} \\
\hline Ave. & 2047.1 & 1944.1 & 1817 & \multirow[t]{3}{*}{11.2} \\
\hline Max. & 2070.6 & 2004.8 & 1830.2 & \\
\hline $\begin{array}{c}\text { Min. } \\
\varepsilon(\%)\end{array}$ & \multicolumn{3}{|c|}{$\varepsilon(\%)$} & \\
\hline Ave. & 9.0 & 8.0 & 8.4 & \multirow[t]{3}{*}{11.1} \\
\hline Max. & 10.8 & 8.7 & 8.6 & \\
\hline Min. & 8.0 & 7.3 & 8.0 & \\
\hline \multicolumn{5}{|l|}{ Flexural } \\
\hline \multicolumn{5}{|l|}{ UFS (MPa) } \\
\hline Ave. & 60.3 & 63.7 & 57.7 & \multirow[t]{3}{*}{9.4} \\
\hline Max. & 67.4 & 65.2 & 58.8 & \\
\hline Min. & 45.1 & 60.6 & 56 & \\
\hline \multicolumn{5}{|l|}{$E_{\mathrm{F}}(\mathrm{MPa})$} \\
\hline Ave. & 1104.5 & 1133.2 & 1053.7 & \multirow[t]{3}{*}{7.0} \\
\hline Max. & 1225.34 & 1181.8 & 1079.2 & \\
\hline Min. & 857.2 & 1008.4 & 1024.9 & \\
\hline \multirow{2}{*}{\multicolumn{5}{|c|}{$\begin{array}{c}\text { Compression } \\
\sigma_{\mathrm{c}}(\mathrm{MPa})\end{array}$}} \\
\hline & & & & \\
\hline Ave. & 54.13 & 53.27 & 52.26 & \multirow[t]{3}{*}{3.4} \\
\hline Max. & 55.82 & 54.95 & 56.02 & \\
\hline Min. & 52.65 & 51.09 & 49.93 & \\
\hline \multicolumn{5}{|l|}{$E_{\mathrm{c}}(\mathrm{MPa})$} \\
\hline Ave. & 741 & 702 & 641 & \multirow[t]{3}{*}{13.4} \\
\hline Max. & 751 & 711 & 649 & \\
\hline Min. & 703 & 687 & 608 & \\
\hline
\end{tabular}

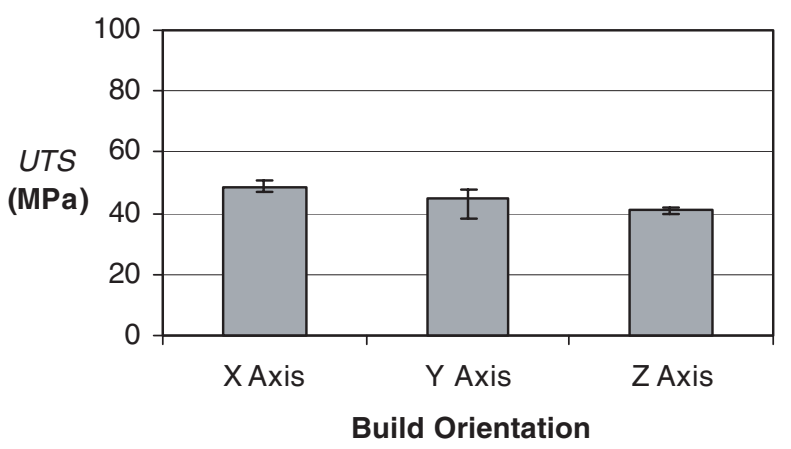

Fig. 8 UTS versus build orientation

tensile test parts which were built in the $x$ axis orientation produced the highest UTS and $E_{\mathrm{T}}$ values, while the lowest values were obtained from parts built in the $z$ axis orientation. The trend continues with the $\varepsilon$ values, which also show that the best tensile test parts are those built in the $x$ axis orientation, although the lowest $\varepsilon$ values come from those produced in the $y$ axis orientation.

From Table 2, the UTS values are heavily influenced by the build orientation of the tensile test parts, with a maximum difference of 16 per cent. The $E_{\mathrm{T}}$ and $\varepsilon$ values are not so strongly affected by

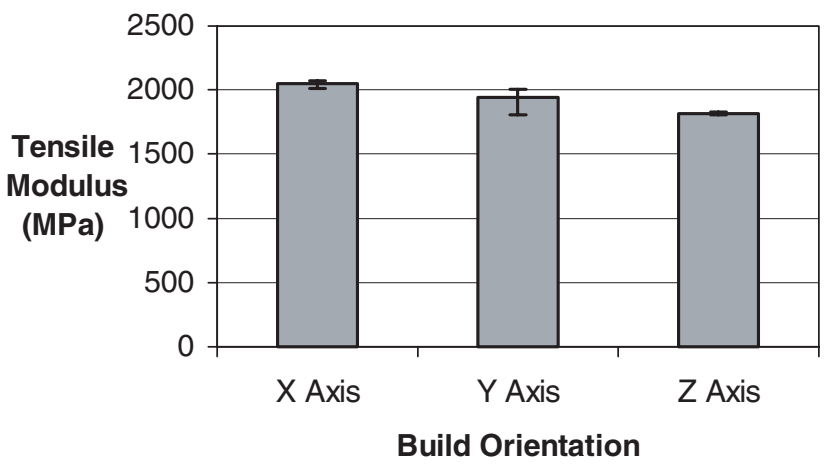

Fig. 9 Tensile modulus versus build orientation

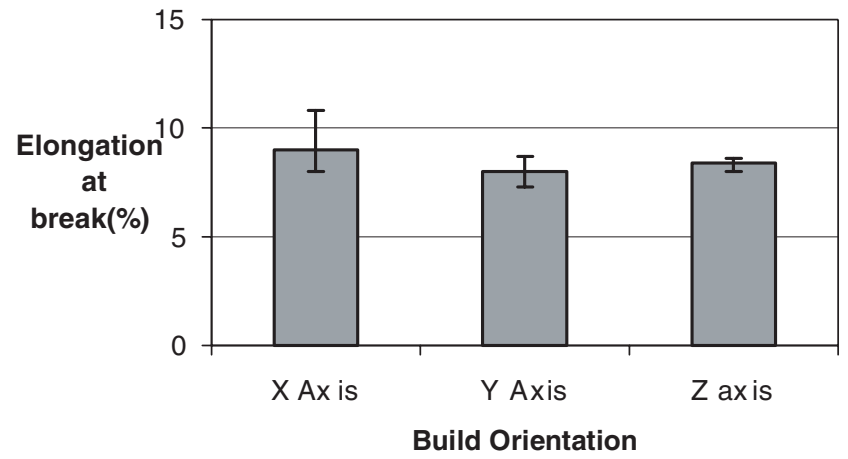

Fig. 10 Elongation at break versus build orientation

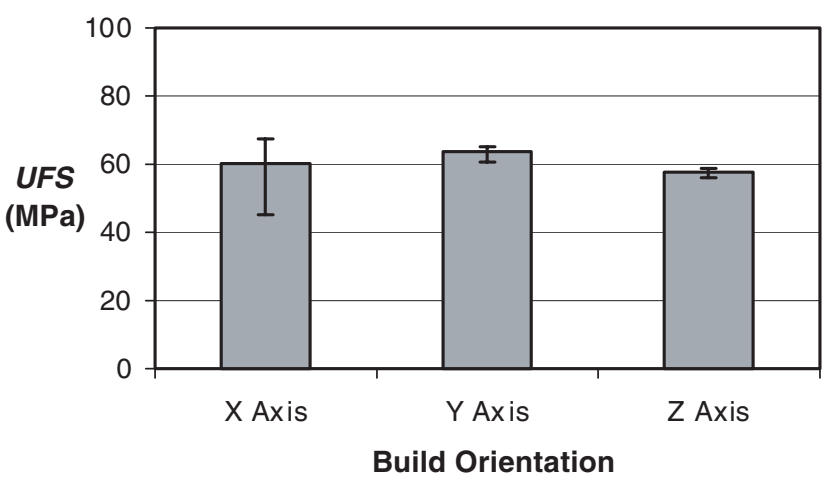

Fig. 11 UFS versus build orientation

the build direction, with only 11.2 per cent and 11.1 per cent respectively, depending on the build orientation of the test part.

\subsection{Flexural properties}

Plots of the UFS and $E_{\mathrm{F}}$ values against their build orientations are shown in Figs 11 and 12. The flexural test results show that the flexural test parts built in the $y$ axis orientation produced the highest UFS and $E_{\mathrm{F}}$ values, while the flexural test parts built in the $z$ axis orientation showed the lowest UFS and $E_{\mathrm{F}}$ values.

From Table 2, the UFS values are dependent on the build orientation of the flexural test parts, with 


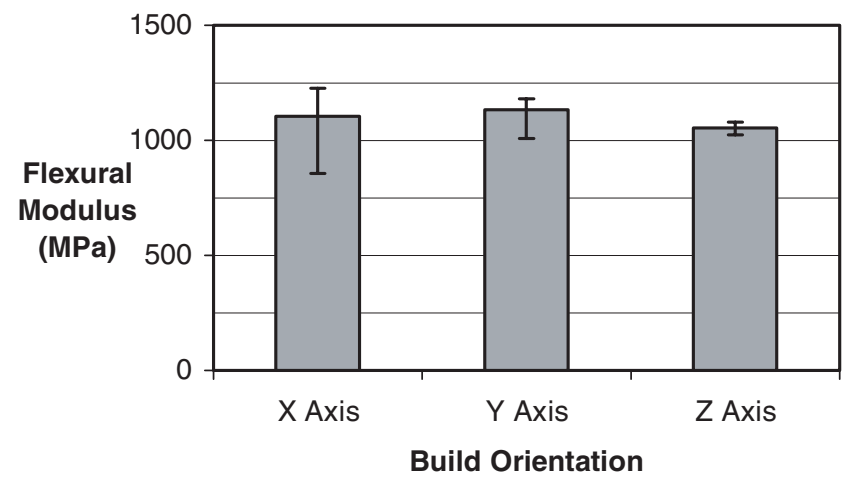

Fig. 12 Flexural modulus versus build orientation

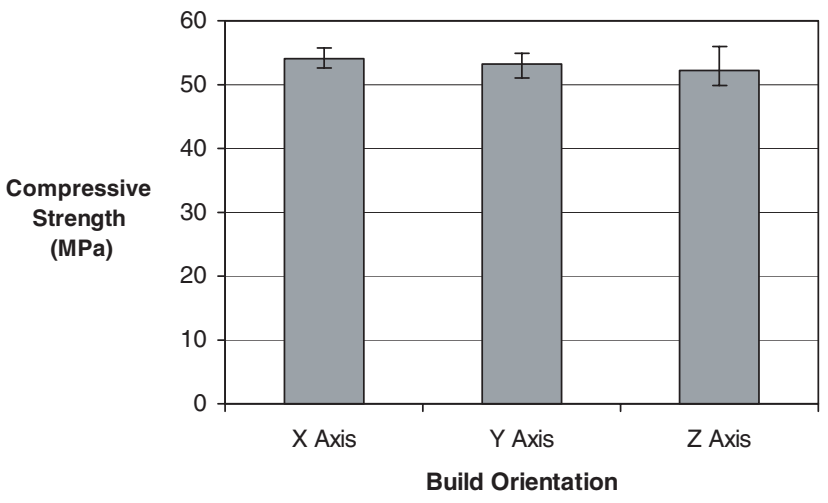

Fig. 13 Compression strength versus build orientation

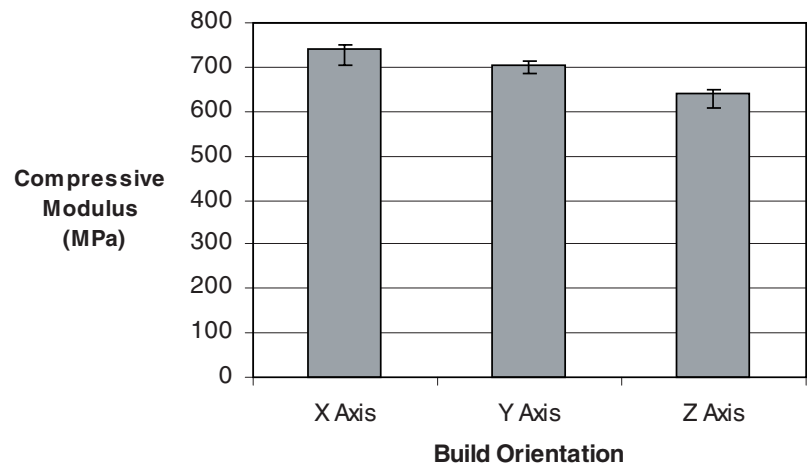

Fig. 14 Compression modulus versus build orientation

a difference of 9.4 per cent. With 7 per cent, $E_{\mathrm{F}}$ values are not as dependent on the build orientation of the test parts.

\subsection{Compressive properties}

The compression tests results are presented in Figs 13 and 14. These show graphs of the $\sigma_{\mathrm{c}}$ and $E_{\mathrm{C}}$ values obtained respectively, against their build orientations. The compression test parts built in the $x$ axis orientation show the highest $\sigma_{\mathrm{c}}$ and $E_{\mathrm{C}}$ values, while the test parts built in the $z$ axis orientation show the lowest $\sigma_{\mathrm{c}}$ and $E_{\mathrm{C}}$ values.
Box-and-Whisker Plot

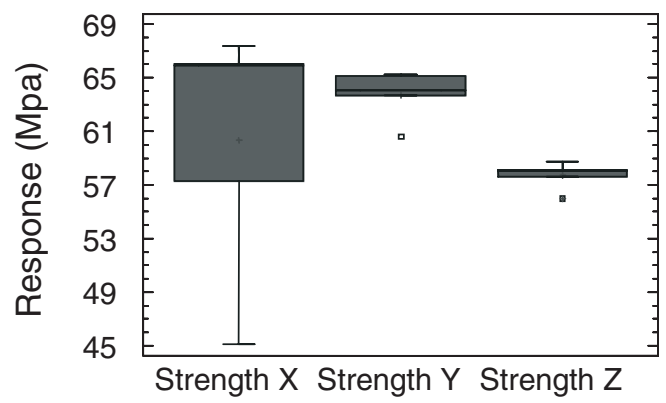

Fig. 15 'Box and whisker' plot of flexural strength

From Table 2, the $\sigma_{\mathrm{c}}$ values show little influence from the build orientation of the compressive test parts, with a maximum difference of 3.4 per cent, while the $E_{\mathrm{C}}$ values are more dependent on the build orientation with a 13.4 per cent maximum difference.

\subsection{Statistical analysis}

Using the results shown in Table 2, a multisample statistical analysis was conducted with the software, Statgraphics. Comparisons were made between the build orientations $(x-y, x-z$ and $y-z)$, within each test type and the confidence level was set to 99.9 per cent. The statistical analysis showed the following.

1. In the majority of cases, different sets of results did not belong to different distributions indicating broadly isotropic behaviour. This is due to the overlap of some of the ranges within the results. An example of this can be seen in Fig. 11, where the range of the $x$ axis result engulfs the ranges of the $y$ and $z$ axes. A typical 'box and whisker' plot (Fig. 15) obtained from the statistical analysis confirms isotropy due to an overlap of the ranges.

2. Anisotropy (i.e. results that come from different distributions) was only observed when comparing $z$ results with either $x$ or $y$ results. This was observed in Fig. 9 (for example), where the range of its $x$ axis does not overlap with its $z$ axis range. A 'box and whisker' plot from the statistical analysis (Fig. 16) showed that there is a significant difference between both sets of data, to confirm the presence of anisotropy. This would be expected in some layer manufactured parts. A full list of the orientations that showed distinct anisotropy among the test parts following the statistical analysis can be seen in Table 3 .

3 . In all cases, the $x$ axis results were shown to come from the same distribution as the $y$ axis results. This is a little surprising, given the sequence of sintering using $x$ direction scanning only; 


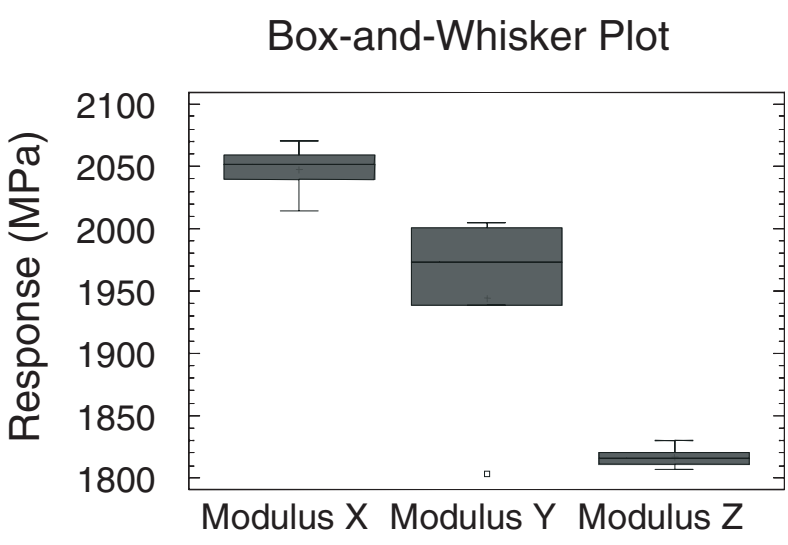

Fig. 16 'Box and whisker' plot of tensile modulus

Table 3 Anisotropy within test parts

\begin{tabular}{lll}
\hline $\begin{array}{l}\text { Mechanical } \\
\text { property }\end{array}$ & Contrast & $\begin{array}{l}\text { Statistically } \\
\text { significant } \\
\text { anisotropy }\end{array}$ \\
\hline Tensile & Strength $x$-strength $z$ & Yes \\
Compression & Modulus $x$-modulus $z$ & Yes \\
& Modulus $x$-modulus $z$ & Yes \\
& Modulus $y$-modulus $z$ & Yes
\end{tabular}

however, all $y$ axis results were obtained from parts that are susceptible to the EOV effect that results in higher (i.e. closer to $x$ axis) mechanical properties.

\section{DISCUSSION}

\subsection{Tensile and compression tests results}

From the results obtained, the tensile and compression test parts built in the $x$ axis orientation produced the highest results. This is due to the strong particle-particle bonding that occurs in the $x$ axis, and the direction of load applied. When a load was applied to a test part built in the $x$ axis, it was parallel to the bonding that occurred during sintering within the part (i.e. layer-layer bonding). As a result of a greater degree of sintering occurring in the $x$ axis and the direction of loading, better mechanical properties are obtained. For test parts built in the $y$ and $z$ axes, the load was applied perpendicular to the layer-layer bonding. The direction of loading, along with a smaller degree of sintering in the $y$ and $z$ axes, resulted in poorer mechanical properties being obtained.

\subsection{Flexural tests results}

The flexural tests show that the highest results obtained came from the test parts built in the $y$ axis

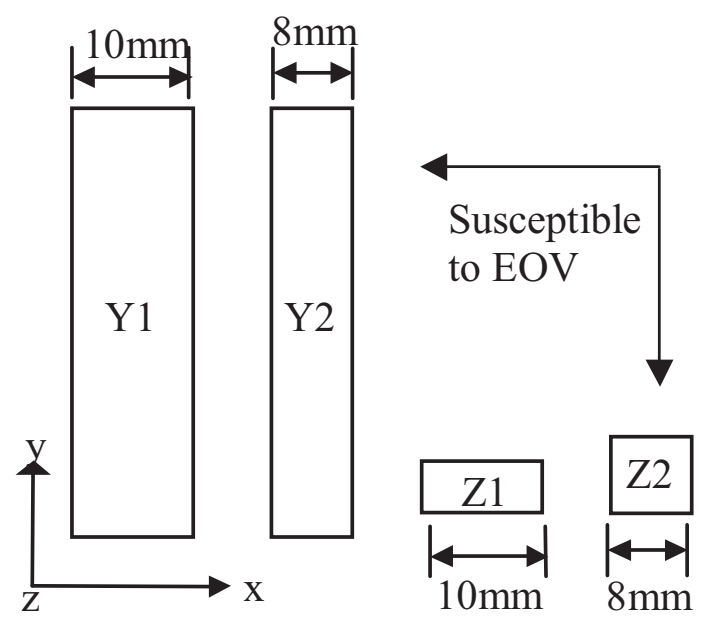

Fig. 17 Top view of flexural test pieces in the $y$ and $z$ axes

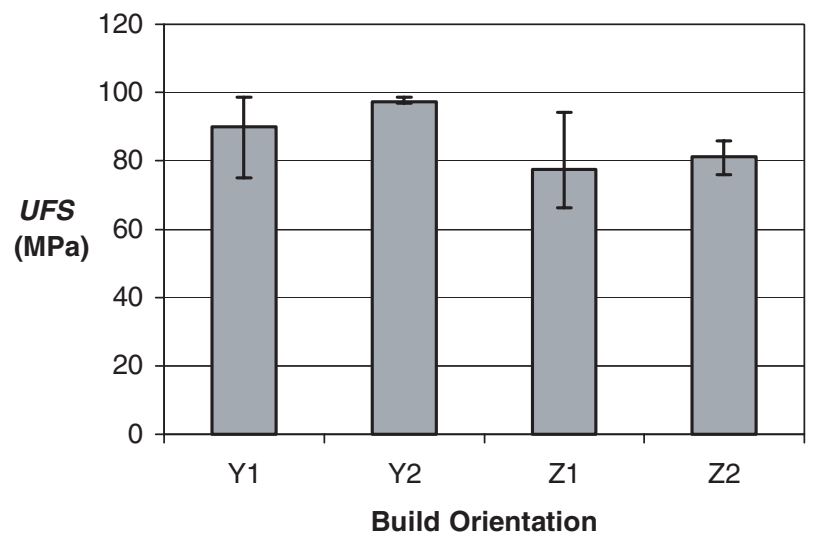

Fig. 18 EOV effect on the UFS of flexural test parts

orientation. The flexural test is the only one in which the loading is applied perpendicularly. In this study, the resultant mechanical properties obtained from flexural test parts built in the $y$ axis orientation are attributed to the EOV effect. To demonstrate this, five flexural test parts were built in each of two orientations along the $y$ and $z$ planes. For easy identification, the flexural test parts with a $10 \mathrm{~mm}$ width were labelled $\mathrm{Y} 1$ and $\mathrm{Z} 1$ respectively, while the flexural test parts with an $8 \mathrm{~mm}$ width were labelled Y2 and Z2 respectively. This is shown in Fig. 17.

It was anticipated that the flexural parts with a smaller $x$ dimension of $8 \mathrm{~mm}$ (Y2 and Z2) would exhibit higher mechanical properties that those with the larger $x$ dimension of $10 \mathrm{~mm}$ (Y1 and Z1). The results obtained are shown in Figs 18 and 19 and they show that, as anticipated, owing to smaller widths, the EOV effect is more prominent in test parts built in the Y2 and Z2 orientations. They have higher strength and modulus values when compared with the Y1 and Z1 parts respectively. 


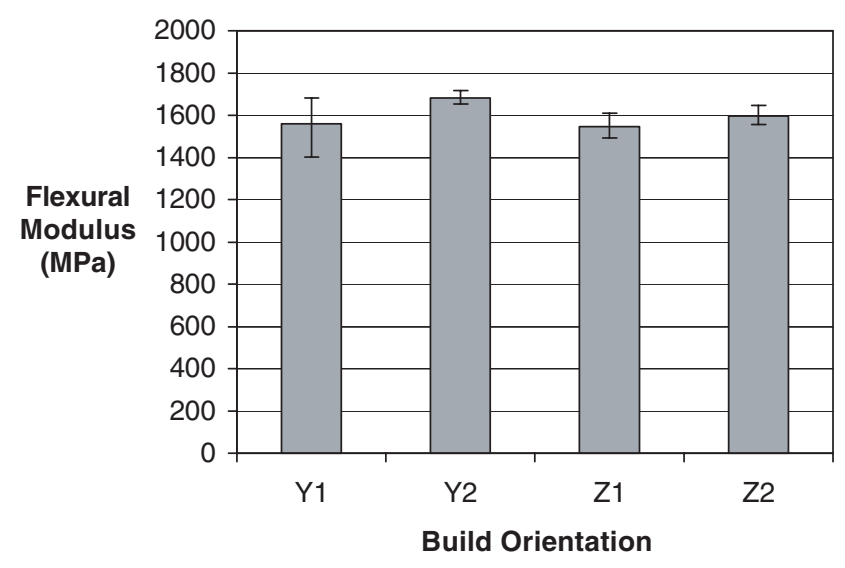

Fig. 19 EOV effect on the flexural modulus of flexural test parts

\subsection{Repeatability in the $z$ axis}

The test parts built in the $z$ axis orientation show the most consistency across all types of test performed, in spite of the fact that $\mathrm{Z}$ generally resulted in the lowest average values. This is an interesting and somewhat surprising result. However, it may be due to the fact that the application of each new layer of powder, with a nominal temperature of $110^{\circ} \mathrm{C}$, from the powder feed bed has a repeatable cooling effect on the underlying fused polymer. This cooling effect will be detrimental to bonding between layers but it is more repeatable than bonding between adjacent particles within a layer that are subject to variations from issues such as end-of-vector, as described above.

\subsection{Anisotropy}

As a result of building the test parts in three different orientations with a laser beam that sinters in only the $x$ axis direction, LS nylon-12 parts show a varying degree of anisotropic material property behaviour. Plastics that are extruded, injection-moulded, or rolled tend to develop an orientation in the processing flow owing to molecular chain alignment. As a result, they develop different properties in the machine and transverse directions [22]. Although LS parts exhibit similar anisotropy, this is not as a result of molecular alignment but as a result of the varying strengths and weaknesses within the part that arise due to the variable temperature changes during the process. The test results in Table 2 show that building parts in different orientations will affect its mechanical properties. The tensile and compressive test parts built in the $x$ axis orientation show the highest results, while the parts built in the $z$ axis orientation show the poorest results. This is in agreement with the results obtained by Gibson and Shi [15] who obtained the best tensile strength results from test parts built in the $x$ axis orientation. Statistically, anisotropy was observed between $x$ and $z$ results for modulus and strength (tensile tests only), as would be expected for a layer manufacturing process. Owing to the low height of the $x$ and $y$ parts, they spend more time after sintering is complete in the hot-build bed than the $z$ parts. This could allow them to continue solid-state sintering after laser-(liquid-state) sintering. Consequently, $x$ and $y$ parts have better properties and more similar properties to each other than the $z$ parts. The flexural test parts also show anisotropy, with the best results coming from the test parts built in the $y$ axis orientation (attributed to EOV effect) and the poorest results coming from parts built in the $z$ axis orientation. This shows how vital it is to consider what mechanical properties are most important before building LS nylon-12 parts that will serve as an end-use product.

\section{CONCLUSION}

The experimental results show evidence of anisotropy in LS-produced parts. This behaviour occurs due to the build orientation of the components and different temperature changes which occur within the parts during the LS process. Tensile and compression test results show that the parts built in the $x$ axis orientation produced the highest mechanical properties, while those built in the $z$ axis orientation produced the least mechanical property values for LS nylon-12. The results obtained from parts built in the $z$ axis orientation were due to weak layer-layer bonding. This offered little resistance to the load applied, which was perpendicular to the layer-layer bonding. This is in contrast with the test parts built in the $x$ axis orientation, where the loading was applied parallel to the layer-layer bonding. Statistical analysis revealed anisotropy in some instances when the $x$ and $y$ orientated parts were each compared to the $z$ orientated parts, but the comparison between the $x$ and $y$ orientated parts showed isotropy with respect to each other in all cases.

The flexural test results showed a different trend. Although the test parts built in the $z$ axis orientation showed the least mechanical property values, the parts built in the $y$ axis orientation showed the highest mechanical property values. This is as a result of the EOV effect, which causes greater sintering and increased densification in flexural parts built in the $y$ axis orientation.

This work has shown that the orientation of a part in a 3D Systems Vanguard SI laser sintering machine is the primary factor affecting its mechanical properties and the EOV effect is the secondary factor 
that is more prominent in parts with small $x$ dimensions.

\section{REFERENCES}

1 Kruth, J. P., Wang, X., Laoui, T., and Froyen, L. Lasers and materials in selective laser sintering. Assembly Automn, 2003, 23(4), 357-371.

2 Hopkinson, N., Hague, R., and Dickens, P. Rapid manufacturing: an industrial revolution for the digital age, 2006 (John Wiley \& Sons Ltd, England).

3 Grimm, T. Rapid prototyping, 2004 (Society of Manufacturing Engineers (SME), Michigan).

4 Greco, A. and Maffezzoli, A. Polymer melting and powder polymer sintering by thermal analysis. J. Thermal Analysis and Calorimetry, 2003, 72, 1167-1174.

5 Mazur, S. Coalescence of polymer particles. In Polymer powder technology (Eds M. Narkis and N. Rosenzweig) 1995, pp. 157-214 (Wiley, Chichester).

6 Philip, J., Bonakdar, L., Poulin, P., Bibette, J., and Calderon-Leal, F. Viscous sintering phenomena in liquid-liquid dispersions. Am. Phys. Soc., 2000, 84(9), 2018-2021.

7 Shi, Y., Li, Z., Sun, H., Huang, S., and Zeng, F. Effect of the properties of the polymer materials on the quality of selective laser sintering parts. Proc. Instn Mech. Engrs, Part L: J. Materials: Design and Applications, 2004, 218(3), 247-252.

8 Frenkel, J. Viscous flow of crystalline bodies under the action of surface tension. J. Phys., 1945, 9(5), 358-391.

9 Scherer, G. W. Sintering of low density glasses: I theory. J. Am. Ceramic Soc., 1977, 60(5-6), 236-239.

10 Scherer, G. W. Viscous sintering under a uniaxial load. J. Am. Ceramic Soc., 1986, 69(9), 206-207.

11 Sun, M., Nelson, J. C., Beaman, J. J., and Barlow, J. W. A model for partial viscous sintering. In Solid freeform fabrication symposium, 1991, pp. 46-55 (The University of Austin, Texas).

12 Pham, D. T., Dimov, S. S., and Lacan, F. Selective laser sintering: applications and technological capabilities. Proc. Instn Mech. Engrs, Part B: J. Engineering Manufacture, 1999, 213(5), 435-449.

13 Dimov, S. S., Pham, D. T., Lacan, F., and Dotchev, K. D. Rapid tooling applications of the selective laser sintering process. Assembly Automn, 2001, 21 (4), 296-302.
14 Hopkinson, N. and Dickens, P. Analysis of rapid manufacturing - using layer manufacturing processes for production. Proc. Instn Mech. Engrs, Part C: J. Mechanical Engineering Science, 2003, 217(C1), 31-39.

15 Gibson, I. and Shi, D. Material properties and fabrication parameters in selective laser sintering process. Rapid Prototyping J., 1997, 3(4), 129-136.

16 Zarringhalam, H. and Hopkinson, N. Post processing of duraform nylon SLS parts for tensile and impact strength improvement. In Solid Freeform Fabrication Symposium, 2003, pp. 596-606 (The University of Austin, Texas).

17 Ho, H. C. H., Gibson, I., and Cheung, W. L. Effects of energy density on morphology and properties of selective laser sintered polycarbonate. J. Mater. Processing Technol., 1999, 89-90, 204-210.

18 Tontowi, A. E. and Childs, T. H. C. Density prediction of crystalline polymer sintered parts at various powder bed temperatures. Rapid Prototyping J., 2001, 7(3), $180-184$

19 ISO 527, Plastics - determination of tensile properties, Parts 1 and 2, 1996 (BSI Publications, London).

20 ISO 178, Plastics - determination of flexural properties, 2001 (BSI Publications, London).

21 ISO 604, Plastics - determination of compressive properties, 2003 (BSI Publications, London).

22 Rosato, D. V., Rosato, D. V., and Rosato, M. G. Plastic design handbook. 2001 (Kluwer Academic Publishers, Massachusetts).

\section{APPENDIX}

\section{Notation}

$E_{\mathrm{c}} \quad$ Young's modulus from compression test (MPa)

$E_{\mathrm{F}} \quad$ Young's modulus from flexural test (MPa)

$E_{\mathrm{T}} \quad$ Young's modulus from tensile test (MPa)

UFS ultimate flexural strength (MPa)

UTS ultimate tensile strength $(\mathrm{MPa})$

$\varepsilon \quad$ elongation at break (\%)

$\sigma_{\mathrm{c}}$ compressive strength (MPa) 